\title{
Effect of standing on ventricular parasystole: shortening of the parasystolic cycle length
}

\author{
Shinji Kinoshita, Takao Mitsuoka
}

\begin{abstract}
Objective-To investigate the effect of standing on the parasystolic cycle length in cases of "true" ventricular parasystole. Methods-Parasystolic cycle length and sinus cycle length were measured during lying and standing in eight men with true ventricular parasystole. These cycle lengths were also measured after exercise in the lying position.

Results-In all cases, parasystolic cycle length and sinus cycle length both shortened on standing, by a mean of $6.4 \%$ and $17 \cdot 8 \%$, respectively, compared to lying. In all cases, the rate of shortening of the parasystolic cycle length was less than that of the sinus cycle length. Parasystolic cycle length was prolonged after exercise, in contrast to a shortening of the sinus cycle length.

Conclusions-Influences on the parasystolic cycle length are not always in the same direction as on the sinus cycle length. This suggests that the effect of autonomic changes on parasystolic rhythm is not always parallel to that on sinus rhythm.
\end{abstract}

(Heart 1997;77:133-137)

Keywords: ventricular parasystole; sinus cycle length; standing; exercise

It has been reported that ventricular premature complexes may often be governed by parasystole and not by ordinary extrasystolic rhythm, particularly in the majority of cases of ventricular bigeminy. ${ }^{1-3}$ However, it is not easy to distinguish between parasystole and ordinary extrasystolic rhythm ${ }^{4-6}$ because it has been shown that in most cases parasystolic rhythm is not regular but depends to a large extent on sinus rhythm..$^{7-9}$ In our previous study, ${ }^{10}$ we emphasised that parasystole should be investigated in cases of "true" parasystole in which one or more "pure" parasystolic cycles containing no intervening non-ectopic

Recently, Kinoshita et al ${ }^{11}$ showed that when an electrocardiogram was taken after exercise in 11 cases of true ventricular parasystole, parasystolic cycle length was considerably prolonged in all cases, in contrast to a shortening of the sinus cycle length. The sinus cycle length also shortened during standing compared with lying. To explore whether or not similar relations in cycle length between parasystole and sinus rhythm are found during standing, the effect of standing on the parasystolic cycle length was investigated in eight cases of true ventricular parasystole.

\section{Methods}

Eight men with true ventricular parasystole were selected for study. In three, pure parasystolic cycles containing no intervening non-ectopic QRS complexes occurred spontaneously (cases 1,4 , and 6), while in five they occurred during temporary sinus arrest caused by vagal stimulation (cases $2,3,5,7$, and 8 ). The basic rhythm was sinus rhythm in all the cases. None of the men had organic heart disease or was receiving antiarrhythmic treatment. Electrocardiograms were recorded after five minutes or more in the lying (supine) position and later while standing. We measured parasystolic cycle length and the sinus cycle length (mean value in the portion containing the parasystolic cycle) in the lying position immediately before standing, and then in the standing position about two minutes of standing. In these cases, exercise was also performed by 50 knee bends. As in our previous study, ${ }^{11}$ when we found the first parasystolic cycle $\mathrm{XX}$ of the long form containing one or two sinus $\mathrm{QRS}$ complexes $\mathrm{R}$ (that is, $\mathrm{XRX}$ or XRRX) after exercise, we measured parasystolic cycle length and sinus cycle length in the lying position.

\section{Results}

The table shows ventricular parasystolic cycle lengths and sinus cycle lengths in the lying and standing positions in eight cases of true parasystole. Cycle lengths are expressed in seconds. In all cases, the parasystolic cycle length and the sinus cycle length both shortened during standing, compared with the values during lying. Parasystolic cycle length in the standing position was shorter than in the lying position by $0.07-0.21 \mathrm{~s}$ (mean $0.12 \mathrm{~s}$ ) or by $2 \cdot 6-9 \cdot 7 \%$ (mean $6 \cdot 4 \%$ ). On the other hand, sinus cycle length in the standing position was shorter than in the lying position by $0.08-0.34 \mathrm{~s}$ (mean $0.19 \mathrm{~s}$ ) second or by $8 \cdot 8-28 \cdot 6 \%$ (mean $17 \cdot 8 \%$ ). In all cases, the rate of shortening in the parasystolic cycle length was smaller than in the sinus cycle length.

In the table, parasystolic cycle lengths and sinus cycle lengths after exercise in six cases (Nos 1-5 and 8) are also shown, measured in the lying position. In all these cases, parasysQRS complexes are found.

Health Administration
Centre, Hokkaido
University, Sapporo,
Japan
S Kinoshita
Department of
Medicine, Taiki Town
Hospital, Taiki,
Hokkaido, Japan
T Mitsuoka
Correspondence to:
Dr Shinji Kinoshita, 4-Jo
3-Chome 3-27, Tonden
Kita-Ku, Sapporo 001,
Japan.
Accepted for publication
16 October 1996


Ventricular parasystolic cycle lengths and sinus cycle lengths in the lying and the standing position in eight male patients

\begin{tabular}{|c|c|c|c|c|c|c|c|}
\hline & \multirow[b]{2}{*}{$\begin{array}{l}\text { Age } \\
\text { (years) }\end{array}$} & \multicolumn{3}{|c|}{ Parasystolic $C L$ (s) } & \multicolumn{3}{|c|}{ Sinus $C L(s)$} \\
\hline & & Lying & Standing & $\begin{array}{l}\text { After } \\
\text { exercise }\end{array}$ & Lying & Standing & $\begin{array}{l}\text { After } \\
\text { exercise }\end{array}$ \\
\hline $\begin{array}{l}\text { Case 1 } \\
\text { Case 2 } \\
\text { Case 3 } \\
\text { Case 4 } \\
\text { Case 5 } \\
\text { Case 6 } \\
\text { Case 7 } \\
\text { Case 8 }\end{array}$ & $\begin{array}{l}36 \\
49 \\
19 \\
22 \\
42 \\
18 \\
25 \\
31\end{array}$ & $\begin{array}{l}1.56 \\
2.17 \\
1.45 \\
1.66 \\
2.73 \\
1.97 \\
2.24 \\
1.55\end{array}$ & $\begin{array}{l}1.48 \\
1.96 \\
1.34 \\
1.57 \\
2.66 \\
1.82 \\
2.07 \\
1.46\end{array}$ & $\begin{array}{l}1.74 \\
2.40 \\
1.51 \\
1.84 \\
2.91 \\
- \\
\overline{1} .64\end{array}$ & $\begin{array}{l}1 \cdot 14 \\
0.80 \\
0.78 \\
0.92 \\
1 \cdot 19 \\
1.09 \\
1.25 \\
1 \cdot 15\end{array}$ & $\begin{array}{l}0.83 \\
0.72 \\
0.68 \\
0.68 \\
0.85 \\
0.95 \\
1.14 \\
0.97\end{array}$ & $\begin{array}{l}0.89 \\
0.62 \\
0.69 \\
0.80 \\
0.75 \\
- \\
\overline{0.89}\end{array}$ \\
\hline
\end{tabular}

$\mathrm{CL}$, cycle length.

Figure 1 Shortening of the parasystolic cycle lengths during standing (case 1). The first three strips were recorded in the lying position immediately before standing and are continuous. The fourth and fifth strips were recorded after about two minutes of standing, and are also continuous. The sixth strip was recorded in the lying position again. The bottom strip was recorded soon after the end of exercise. Time intervals are expressed in hundredths of a second. Numerals marked with an asterisk indicate lengths of pure parasystolic cycles containing no intervening non-ectopic $Q R S$ complexes. tolic cycle length was prolonged, in contrast to shortening of the sinus cycle length, as in our previous study. ${ }^{11}$ Parasystolic cycle length after exercise was longer than before exercise by $0.06-0.23 \mathrm{~s}$ (mean $0.15 \mathrm{~s}$ ) or by $4.1-11.5 \%$ (mean $8 \cdot 2 \%$ ). Sinus cycle length after exercise was shorter than before exercise by $0.09-0.44 \mathrm{~s}$ (mean $0.22 \mathrm{~s}$ ) or by $11.5-37.0 \%$ (mean $21 \cdot 4 \%$ ). In cases 6 and 7, parasystolic cycle length after exercise is not shown in the table because we were unable to find a parasystolic cycle XX containing one or two sinus QRS complexes $\mathrm{R}$ (XR or XRRX) after exercise.

Figure 1 shows a typical case (case 1). The first three strips were recorded with the patient lying down immediately before standing, and are continuous. The top strip shows an ectopic cycle XRX of $1.56 \mathrm{~s}$ containing an early intervening sinus $Q R S$ complex $R$. Time intervals are expressed in hundredths of a second. In the second and third strips, pure ectopic cycles of the same length $\mathrm{XF}$ and $\mathrm{XX}$ are found which contain no intervening non-ectopic
Standing position
QRS complexes. This indicates that this case is "true" parasystole.

In the second strip, a long interectopic interval XR'RX of $2.92 \mathrm{~s}$ is considerably shorter than twice the pure parasystolic cycle length of $1.56 \mathrm{~s}$. In the interval $\mathrm{XR}^{\prime} \mathrm{RX}$, the sinus QRS complex $R^{\prime}$ occurs comparatively late in the parasystolic cycle. This suggests that the late sinus impulse hastens the subsequent discharge $(\mathrm{X})$ of the ectopic focus. ${ }^{12-14}$ Hastening of the subsequent ectopic discharge by such a late sinus QRS complex $R^{\prime}$ occurs also in the other portions of fig 1 . In the figure, $\mathrm{X}$ and $(\mathrm{X})$ represent manifest and concealed ectopic depolarisations, respectively. F represents a fusion $Q R S$ complex. $R$ and $R^{\prime}$ represent sinus QRS complexes that occur comparatively early and late in the parasystolic cycle, respectively. Numerals marked with an asterisk indicate lengths of pure parasystolic cycles.

The fourth and fifth strips were recorded in the standing position after about two minutes of standing, and are continuous. The length of the parasystolic cycle XRX is $1.48 \mathrm{~s}$ in the strips. This indicates that the parasystolic cycle length during standing is considerably shorter than during lying $(1.56 \mathrm{~s})$. The sinus cycle length in these strips is $0.83 \mathrm{~s}$ on average. Thus the sinus cycle length during standing is also shorter than during lying $(1 \cdot 14 \mathrm{~s}$ on average), though the rate of shortening in sinus cycle length $(27 \cdot 2 \%)$ is considerably greater than in parasystolic cycle length $(5 \cdot 1 \%)$.

The electrocardiogram was then taken in the lying position again. The sixth strip was
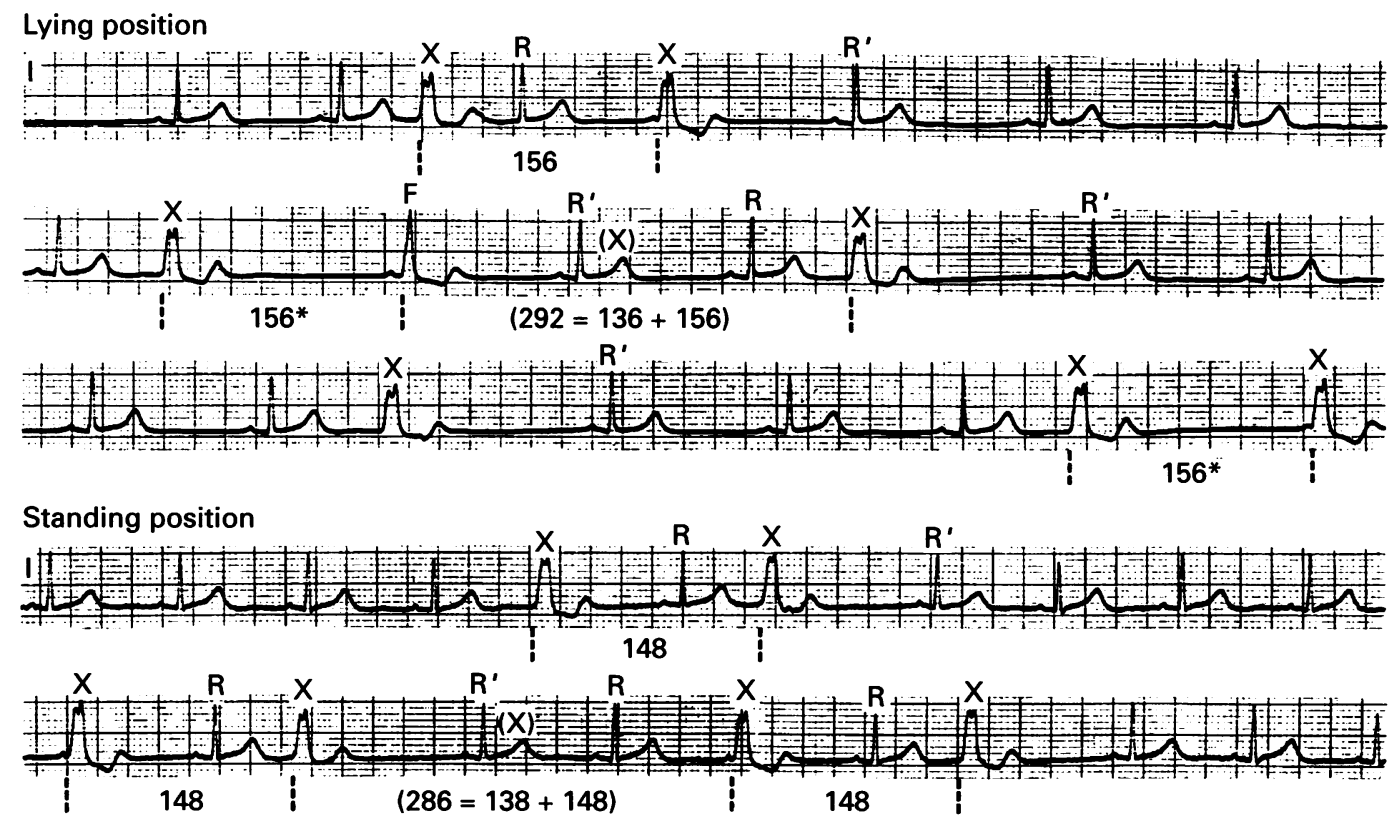

Lying position

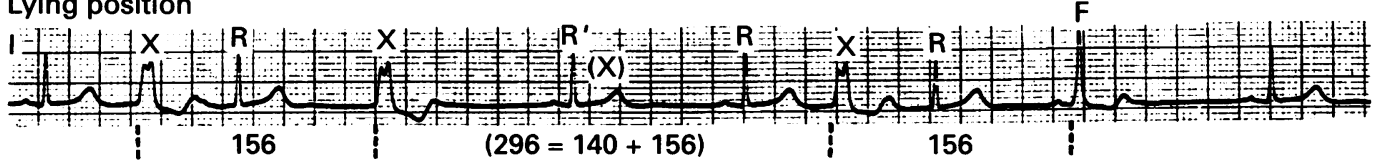

After exercise

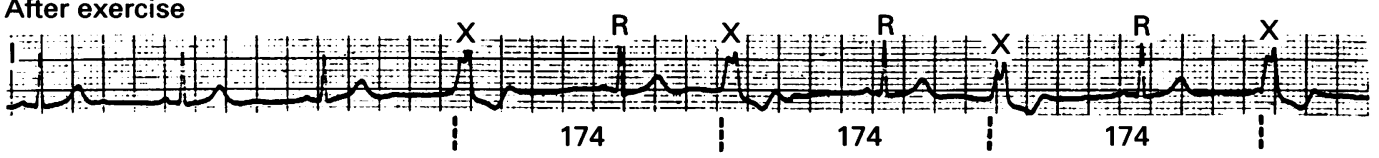


Figure 2 Shortening of the parasystolic cycle length during standing (cases 2 and 3). The upper three strips (case 2) and the lower three strips (case 3) were recorded during lying, during standing, and during temporary sinus arrest caused by vagal stimulation. Parts of the electrocardiograms for case 2 were shown in fig 3 of our previous paper in which a prolonged parasystolic cycle $X R R X$ after exercise was found. $X$ and $(X)$, manifest and concealed parasystolic depolarisations, respectively; $F$, fusion $Q R S$ complex; $R$ and $R^{\prime}$, sinus $Q R S$ complexes that intervene comparatively early and late in the parasystolic cycle, respectively.

Case 2
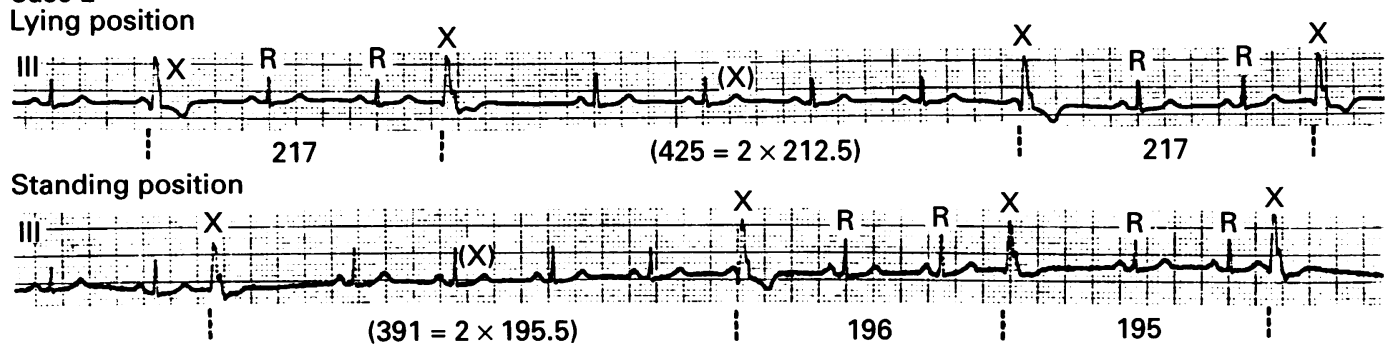

Vagal stimulation

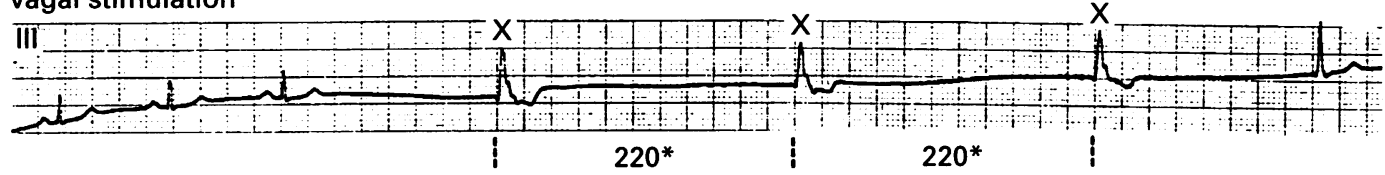

Case 3

Lying position

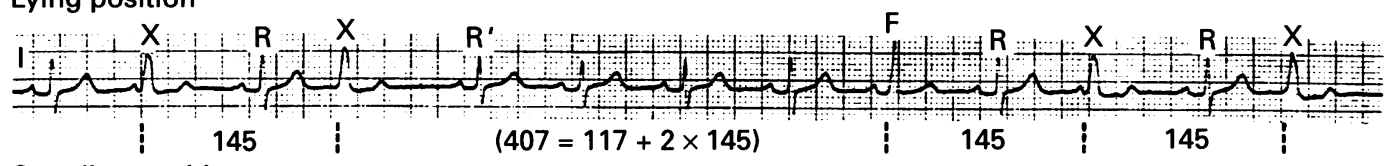

Standing position

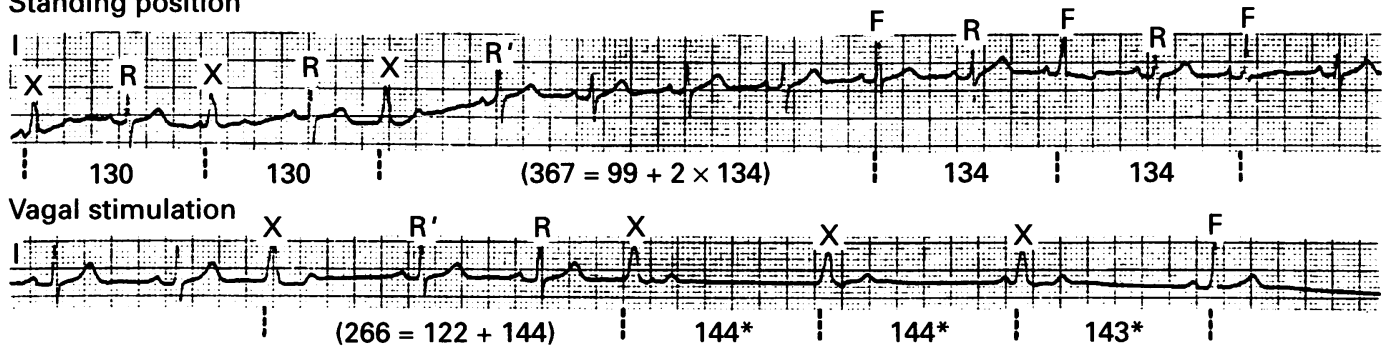

recorded in the lying position about two minutes after lying down. The length of the parasystolic cycle XRX (1.56 s) and of the sinus cycle $(1.14 \mathrm{~s}$ on average) in this strip are the same as in the first three strips. Thus both the cycle lengths return to the values found in the lying position before standing.

Next, exercise was performed by 50 knee bends. The bottom strip was recorded in the lying position soon after the end of exercise. After exercise, the sinus cycle shortens again. Thus the sinus cycle length after exercise $(0.89 \mathrm{~s}$ on average) is shorter than before exercise $(1 \cdot 14 \mathrm{~s})$. On the other hand, the length of the parasystolic cycle XRX (1.74 s) is considerably greater than before exercise $(1.56 \mathrm{~s})$. Such prolongation of parasystolic cycle length after exercise, in contrast to shortening of the sinus cycle length, was also found in our previous study. " Our present findings show that sinus cycle length shortens both during standing and after exercise, whereas parasystolic cycle length shortens during standing and lengthens after exercise. Hastening of the subsequent ectopic discharge by a late sinus QRS complex $R^{\prime}$ as seen in fig 1 also occurred in all other cases except one (case 2 ).

Figure 2 shows two other typical cases (cases 2 and 3). The upper three strips (case 2) and the lower three strips (case 3) were recorded during lying, during standing, and during temporary sinus arrest caused by vagal stimulation from pressure on the eyeball. In both cases, pure ectopic cycles XX containing no intervening non-ectopic QRS complexes are found during temporary sinus arrest caused by vagal stimulation. This shows that these cases are also "true" ventricular parasystole. In these cases, also, both parasystolic cycle length and sinus cycle length shortened during standing. After exercise, however, parasystolic cycle length was prolonged, in contrast to shortening of sinus cycle length, as shown in the table.

The first and second strips (case 2) in fig 2 show that parasystolic cycle length XRRX in the standing position (1.95 or $1.96 \mathrm{~s})$ is shorter than in the lying position $(2.17 \mathrm{~s})$ by $9 \cdot 7 \%$. Sinus cycle length in the standing position ( $0.72 \mathrm{~s}$ on average) is also shorter than in the lying position $(0.80 \mathrm{~s}$ on average) by $10.0 \%$. In this case, the parasystolic cycle length is markedly long, and the parasystolic cycle containing one intervening sinus QRS complex (XRX) is usually not found. Thus, hastening of the subsequent ectopic discharge by a late sinus QRS complex is not clearly found, though there is the possibility that the second intervening sinus QRS complex $R$ of the parasystolic cycle XRRX may slightly hasten the subsequent ectopic discharge.

The fourth and fifth strips (case 3) show that the length of the parasystolic cycle FRF in the standing position ( $1.34 \mathrm{~s})$ is shorter than that of the parasystolic cycle XRX in the lying position $(1.45 \mathrm{~s})$ by $7 \cdot 6 \%$. Sinus cycle length in the standing position $(0.68 \mathrm{~s})$ is also shorter than in the lying position $(0.77 \mathrm{~s})$ by $10 \cdot 8 \%$. In this case, hastening of the subsequent ectopic discharge by a late sinus QRS complex $R$ is clearly seen. In the fifth strip, the parasystolic cycle XRX (1.30 s) is slightly shorter than the cycle FRF ( $1.34 \mathrm{~s})$, possibly because the sinus QRS complex $R$ in the cycle XRX may 
intervene somewhat later than the $\mathrm{R}$ in the cycle FRF.

\section{Discussion}

DIFFERENTIATION OF “TRUE” PARASYSTOLE FROM ORDINARY EXTRASYSTOLIC RHYTHM

It had been believed for a long time that parasystolic rhythm is regular and independent of the basic (usually sinus) rhythm. In 1974, Kinoshita ${ }^{15}$ reported a case of ventricular parasystole in which, when a sinus QRS complex occurred comparatively late in the parasystolic cycle, the subsequent discharge of the parasystolic focus was considerably hastened. Since then, $\mathrm{we}^{16-18}$ and other investigators $^{2819}$ have shown that such hastening of the ectopic discharge occurs in most cases of parasystole. It has been suggested that such "irregular" parasystole is caused by electronic modulation, ${ }^{20-22}$ or by type $\mathrm{I}^{7915}$ or type $\mathrm{II}^{2324}$ second degree entrance block. Thus constant shortest interectopic intervals cannot be used as a diagnostic criterion of parasystole. On the other hand, markedly variable coupling intervals are found in many cases of ordinary ventricular extrasystole that are not governed by parasystole. ${ }^{625}{ }^{26}$ Accordingly, the classical criteria for diagnosis of parasystole (that is, varying coupling intervals and constant shortest interectopic intervals) cannot be used in most cases of parasystole. In our previous studies, ${ }^{41011}$ we emphasised that in order to distinguish between true parasystole and ordinary extrasystolic rhythm, one or more pure ectopic cycles containing no intervening non-ectopic QRS complexes must be found spontaneously or during temporary sinus arrest caused by vagal stimulation. For the present study, cases of such true ventricular parasystole were selected to investigate the effect of standing on the parasystolic cycle.

\section{SHORTENING OF THE PARASYSTOLIC CYCLE}

LENGTH DURING STANDING

The sinus cycle length usually shortens both during standing and after exercise. It seems that shortening of the sinus cycle length after exercise is caused by increased sympathetic tone. The enhancing effect of sympathetic discharge on the ventricular parasystolic cycle length was also reported by Castellanos et al. ${ }^{27}$ However, the findings in our present study show that parasystolic cycle length was prolonged after exercise, whereas it shortened during standing. This indicates that changes of parasystolic cycle length are not always in the same direction as those of sinus cycle length; thus that influence on automaticity in the parasystolic pacemaker is not always parallel to that in the sinus node.

Though the mechanism for this discrepancy between parasystolic rhythm and sinus rhythm is not revealed by the present study, it appears that automaticity in the parasystolic pacemaker may be affected by changes in vagal tone rather than in sympathetic tone as a result of autonomic interactions. ${ }^{28}$ It is thought that during standing, vagal tone is depressed mainly because venous return to the right atrium is decreased. On the other hand, it seems that after exercise, though sympathetic tone is increased, there is also a compensatory increase in vagal tone, mainly because venous return to the right atrium is increased. Automaticity in the parasystolic pacemaker may be affected by such changes in vagal tone rather than by those in sympathetic tone. In our previously reported cases, ${ }^{15} 29$ it was shown that during vagal stimulation by injection of pilocarpine, parasystolic cycle length was definitely prolonged in a way similar to that after exercise. Thus it appears that during standing automaticity may be enhanced both in the sinus node and in the parasystolic pacemaker by decreased vagal tone. On the other hand, after exercise, though automaticity may be enhanced in the sinus node by increased sympathetic tone, it may be depressed in the parasystolic pacemaker by a compensatory increase in vagal tone. It has been reported that during vagal stimulation from carotid pressure, parasystolic cycle length was also prolonged, ${ }^{30}$ though in our present and previous studies ${ }^{11}$ parasystolic cycle length was only slightly prolonged or, in some cases, slightly shortened during vagal stimulation from eyeball pressure. Further investigation seems necessary to clarify the effect of changes in autonomic tone on the parasystolic rhythm.

\section{CONCLUSIONS}

Our findings show that influences on parasystolic cycle length did not always act in the same direction as those on sinus cycle length. This suggests that influences on parasystolic rhythm may be different from those on sinus rhythm or on ordinary ventricular rhythm. Such differences should be borne in mind when patients with ventricular premature complexes are investigated

1 Moe GK, Jalife J, Mueller WJ, Moe B. A mathematica model of parasystole and its application to clinical model of parasystole and its application

2 Oreto G, Luzza F, Satullo G, Schamroth L. Modulated ventricular parasystole as a mechanism for concealed ventricular parasystole as a mechanism
bigeminy. Am $\mathcal{f}$ Cardiol 1986;58:954-8.

3 Nilsson G, Ahlfeldt H, Ahren T, Jonasson T. Distribution patterns of ventricular premature complexes in long-term electrocardiographic recordings and their usefulness in disclosing modulated parasystole. Am f Cardiol 1991, 68:1045-8.

4 Kinoshita S, Konishi G, Okada F. Differentiation between parasystole and reentry in concealed bigeminy. Cardiology 1992;81:100-6.

5 Kinoshita S, Okada F. Mechanism of concealed bigeminy. Am 7 Cardiol 1992;69:976.

6 Kinoshita S, Okada F, Konishi G, Kinoshita M, Ogawa S Bradycardia-dependent and tachycardia-dependent termination of ventricular bigeminy: mechanism of ventricular extrasystoles with fixed coupling. Am Heart $\mathcal{f} 1995$; 129:557-64.

7 Kinoshita S. Mechanisms of ventricular parasystole. Circulation 1978;58:715-22.

8 Tenczer J, Littmann L, Rohla M, Wu DB, Fenyvesi T. A study of modulated ventricular parasystole by programmed stimulation. Am f Cardiol 1987;59:846-5

9 Kinoshita S, Konishi G, Mizutani M, Tanabe Y. Influence of sinus impulses on the parasystolic cycle length. $f$ Electrocardiol 1989;22:285-91.

10 Kinoshita S, Okada F, Konishi G, Kinoshita M, Ito Y. Differentiation between parasystole and extrasystoles influence of vagal stimulation on parasystolic impulse for mation. $\mathcal{F}$ Electrocardiol 1994;27:169-74.

11 Kinoshita S, Ogawa S, Mitsuoka T. Reverse effects of exercise on the parasystolic and sinus cycle lengths. $f$ Electrocardiol 1996;28:131-7.

12 Jalife J, Moe GK. Effect of electrotonic potentials on pacemaker activity of canine Purkinje fibers in relation to parasystole. Circ Res 1976;39:801-8.

13 Kinoshita S. Intermittent parasystole originating in the reentrant path of ventricular extrasystoles. Chest 1977; 72:201-6. 
14 Kinoshita S, Okada F, Konishi G. Mechanism of irregular parasystole: differentiation of second-degree entrance block from electrotonic interaction. Am Heart 7 1992; 124:816-23.

15 Kinoshita S. Wenckebach phenomenon of entrance block in intermittent ventricular parasystole. Chest 1974;66: 530-5.

16 Kinoshita S, Kawasaki T. Ventricular parasystole and couplets: the concept of longitudinal dissociation in the microreentry pathway. Am Heart $f$ 1983;105:1050-5.

17 Kinoshita S, Mizutani M. Effect of sinus impulses on ventricular parasystolic thythm. Am Heart $\mathcal{f}$ 1983;106: tricular

18 Kinoshita S, Konishi G, Kinoshita Y. Protection of the ventricular parasystolic focus due to interference of sinus and parasystolic impulses in the ventricular-ectopic junction. Cardiology 1990;77:66-70.

19 Castellanos A, Luceri RM, Moleiro F, Kayden DS, Trohman RG, Zaman L, et al. Annihilation, Entrainment and modulation of ventricular parasystolic rhythms. $A m \mathcal{F}$ Cardiol 1984;54:317-22. 20 Jalife J, Moe GK. A biological model of parasystole. Am $\mathcal{F}$

21 Antzelevitch C, Jalife J, Moe GK. Characteristics of reflection as a mechanism of reentrant arrhythmias and its relationship to parasystole. Circulation 1980;61:182-91.

22 Jalife J, Antzelevitch C, Moe GK. The case for modulated parasystole. PACE 1982;5:911-26.

23 Kinoshita S, Tanabe Y. Second degree entrance block in intermittent ventricular parasystole. Chest 1975;67: 236-7.

24 Kinoshita S. Mechanisms of intermittent ventricular parasystole due to type II second degree entrance block. $\mathcal{f}$ Electrocardiol 1983;16:7-14.

25 Kinoshita S, Konishi G, Kinoshita Y. Mechanism of ventricular extrasystoles with fixed coupling: a theoretical model derived from the concept of longitudinal dissociation in the reentrant pathway of extrasystoles. $f$ Electrocardiol 1990;23:249-54.

26 Kinoshita S, Konishi G, Kinoshita Y. Intermittent ventricular bigeminy as an expression of two-level Wenckebach periodicity in the reentrant pathway of extrasystoles.
$P A C E$ 1990;13:119-22.

27 Castellanos A, Mendoza IJ, Luceri RM, Castillo CA Zaman L, Saoudi N, et al. Concealment of manifest, and exposure of concealed, ventricular parasystole produced by isoproterenol. Am $\mathcal{f}$ Cardiol 1985;55:1344-9.

$28 \mathrm{Levy}$ MN. Autonomic interactions in cardiac control. In Coumel P, Garfein OB, eds. Electrocardiography: past and future. New York: The New York Academy of Sciences, 1990:209-21.

29 Kinoshita S, Mashimo K. Effect of vagal stimulation on ventricular impulse formation in a case of ventricula parasystole (in Japanese). Shinzo 1969;1:1185-94.

30 Nau GJ, Aldariz AK, Acunzo RS, Halpern MS, Davidenko JM, Elizari MV, Rosenbaum MB. Modulation of parasystolic activity by nonparasystolic beats. Circulation 1982;66:462-9. 